Pacific Journal of Mathematics

A DESCRIPTION OF THE TOPOLOGY ON THE DUAL SPACE 


\title{
A DESCRIPTION OF THE TOPOLOGY ON THE DUAL SPACE OF A NILPOTENT LIE GROUP
}

\author{
KENNETH I. JOY
}

\begin{abstract}
The study of convergence of sequences of elements of the dual space $\hat{G}$, for a nilpotent Lie group $G$, is done by reducing the study to convergence of sequences of subgroup representation pairs, whose subgroup component has dimension less than the dimension of $G$. The main results are then applied to give a new proof to the fact that the Kirillov correspondence is a homeomorphism for nilpotent Lie groups.
\end{abstract}

1. Introduction. Let $G$ be a real, connected, simply-connected nilpotent Lie group. By the dual space $\hat{G}$ of $G$, we mean the set of all equivalence classes of irreducible unitary representations equipped with the hull-Kernel topology. Kirillov [4] has shown that the elements of $\hat{G}$ are in one-to-one correspondence with orbits of real linear functionals on $g$ (the Lie algebra of $G$ ). The fact that this correspondence is actually a homeomorphism was conjectured by Kirillov and first proved by Brown [1].

In this paper we study the convergence of sequences $\left\{W_{n}\right\}$ in $\hat{G}$ by studying sequences of subgroup-representation pairs $\left\{\left(H_{n}, S_{n}\right)\right\}$, where $H_{n}$ is a subgroup of $G, S_{n} \in \hat{H}_{n}$ and $\operatorname{dim} H_{n}<\operatorname{dim} G$. We develop necessary and sufficient conditions for the convergence of sequences in $\hat{G}$, in terms of convergence of associated sequences of subgroup-representation pairs in the subgroup-representation topology of Fell [3]. Then using the theorems of Fell, we give a new proof of the Kirillov conjecture that avoids the use of the free nilpotent Lie algebras of Brown.

2. Preliminaries. Let $G$ be a simply connected nilpotent Lie group. We will be primarily interested in the case when $G$ is nonabelian. For if $G$ were abelian, then $G \cong \mathbf{R}^{n}$ for some $n$, and the irreducible representations of $G$ are characters. In fact, $\hat{\mathbf{R}}^{n} \cong \mathbf{R}^{n}$. Therefore, the case when $G$ is abelian is essentially trivial and, unless otherwise noted, we will assume our groups are nonabelian.

We introduce the following conventions in notation:

(1) $\mathcal{K}(G)$ will denote the set of all closed subgroups of $G$, equipped with the compact-open topology

(2) $\mathscr{Q}(G)$ will denote the set of all subgroup-representation pairs of $G$, equipped with the subgroup-representation pair topology of Fell [3] 
(3) If $g$ is the Lie algebra of $G$, there is a one-to-one correspondence between closed, connected subgroups of $G$ and Lie subalgebras of $g$. If $H$ is a closed, connected subgroup of $G$ we will denote its corresponding subalgebra by $\mathfrak{h}$, and conversely.

(4) We will denote by ind ${ }_{H}^{G} S$ the representation of $G$, induced by the representation $S$ of $H$, and by $\left.W\right|_{H}$ the restriction of the representation $W$ to the subgroup $H$.

(5) By Kirillov [4], the irreducible representations of $G$ are in one-toone correspondence with certain orbits in $\mathfrak{g}^{*}$ (the conjugate space of $\mathfrak{g}$ ). If $W \in \hat{G}$, we will denote its correspondence orbit by $\Omega_{W}$.

3. The topology of $\hat{G}$. Let $G$ be a simply connected, nilpotent Lie group.

3.1 Definition. Let $(H, S) \in \mathbb{Q}(G)$ where $S \in \hat{H}$ and $S$ is not a character. A pair $\left(H^{\prime}, S^{\prime}\right) \in \mathbb{Q}(G)$ is called inducing for $(H, S)$ if $H^{\prime}$ is of codimension one in $H$ and $S=\operatorname{ind}_{H^{\prime}}^{H} S^{\prime}$.

3.2 REMARK. The existence of inducing pairs, when $S$ is not a character, follows from [4].

3.3 LemMa. Let $H$ be a subgroup of $G$ of codimension one, and let $W \in \hat{G}$. Let $f \in \Omega_{W}$ and $T$ be chosen so that $\left.f\right|_{\mathfrak{h}} \in \Omega_{T}$. Then $W \subseteq_{\mathrm{w}} \operatorname{ind}_{H}^{G} T$ and $T \in \operatorname{sp}\left(\left.W\right|_{h}\right)$.

Proof. By [4] the orbit $\Omega_{W}$ can be classified as one of two types. The Lemma then follows directly from Lemma 6.2 of [4] and Theorem 4.3 of [3], or by Lemma 6.3 of [4] and Theorem 4.5 of [3].

We now develop necessary and sufficient conditions for a sequence of subgroup-representation pairs to converge in $\mathscr{Q}(G)$.

3.4 TheOREM. Let $\left\{\left(H_{n}, S_{n}\right)\right\}$ be a sequence in $\mathbb{Q}(G)$ such that, for each $n, S_{n} \in \hat{H}_{n}$ and $S_{n}$ is not a character. Then $\left(H_{n}, S_{n}\right) \rightarrow(H, S)$ in $\mathbb{Q}(G)$ if and only if, for each subsequence of $\left\{\left(H_{n}, S_{n}\right)\right\}$, there exists a subsequence $\left\{\left(H_{i}^{\prime}, S_{i}^{\prime}\right)\right\}$ such that:

(1) there exist inducing pairs $\left(H_{n}^{\prime \prime}, T_{n}\right)$ for each $\left(H_{n}^{\prime}, S_{n}^{\prime}\right)$ such that

$$
\left(H_{n}^{\prime \prime}, T_{n}\right) \rightarrow\left(H^{\prime \prime}, T\right) \quad \text { in } \mathbb{Q}(G)
$$

for some $T \in \hat{H}^{\prime \prime}$.

(2) $S \subseteq \subseteq_{\mathrm{w}}$ ind $_{H^{\prime \prime}}^{H} T$. 
Proof. $(\Rightarrow)$ Let $\left(H_{n}, S_{n}\right) \rightarrow(H, S)$ in $\mathscr{Q}(G)$, and let $\left\{\left(H_{i}^{\prime}, S_{i}^{\prime}\right)\right\}$ be a subsequence of $\left\{\left(H_{n}, S_{n}\right)\right\}$. For each $i$, let $\left(H_{i}^{\prime \prime}, S_{i}^{\prime \prime}\right)$ be an inducing pair for $\left(H_{i}^{\prime}, S_{i}^{\prime}\right)$. By restricting to subsequences, it is sufficient to assume that all the $H_{l}^{\prime \prime}$ have the same dimension. Therefore since $\mathcal{K}(G)$ is compact, by further restricting to a subsequence, we can assume there exists an $H^{\prime \prime} \in \mathcal{K}(G)$ of codimension one in $H$ such that $H_{i}^{\prime \prime} \rightarrow H^{\prime \prime}$ in $\mathcal{K}(G)$.

By continuity of restriction (Theorem 3.2 of [2]) we have

$$
\left(H_{l}^{\prime \prime},\left.S_{i}^{\prime}\right|_{H_{l}^{\prime \prime}}\right) \rightarrow\left(H^{\prime \prime},\left.S\right|_{H^{\prime \prime}}\right) \text { in } \mathbb{Q}(G) \text {. }
$$

Pick $f \in \Omega_{s}$ and $T$ such that $\left.f\right|_{\mathfrak{h}^{\prime \prime}} \in \Omega_{T}$. By Lemma 3.3, $T \in \operatorname{sp}\left(\left.S\right|_{H^{\prime \prime}}\right)$ and $S \subseteq \subseteq_{\mathrm{w}} \operatorname{ind}_{H^{\prime \prime}}^{H} T$. Now $T$ is in the closure of the set

$$
E=\bigcup_{i}\left\{\left(H_{i}^{\prime \prime}, T^{\prime \prime}\right): T^{\prime \prime} \in \operatorname{sp}\left(\left.S_{i}^{\prime}\right|_{H_{i}^{\prime \prime}}\right)\right\}
$$

(Lemma 3.3 of [5]), and the closure of $E$ is the same as the closure of

$$
\bigcup\left\{\left(H_{i}^{\prime \prime}, T^{\prime \prime}\right): T^{\prime \prime} \text { is in the orbit of } H_{i}^{\prime \prime} \text { associated with } S_{l}^{\prime}\right\}
$$

(Theorem 4.5 of [4]). Therefore, for each $i$, there exists $T_{l}$ contained in the orbit of $\hat{H}_{i}^{\prime \prime}$ associated with $S_{i}^{\prime}$ such that $\left(H_{i}^{\prime \prime}, T_{i}\right) \rightarrow(H, T)$ in $\mathcal{Q}(G)$. Since $S_{i}^{\prime \prime}$ and $T_{i}$ must be in the same orbit of $\hat{H}_{i}^{\prime \prime}$ associated with $S_{i}^{\prime}$, we have that $\left(H_{i}^{\prime \prime}, T_{i}\right)$ is inducing for $\left(H_{i}^{\prime}, S_{l}^{\prime}\right)$.

The sequence $\left\{\left(H_{i}^{\prime}, S_{i}^{\prime}\right)\right\}$ and the pair $(H, T)$ satisfy the conditions of the Theorem.

$(\Leftarrow)$ Suppose that for each subsequence of $\left\{\left(H_{n}, S_{n}\right)\right\}$ there exists a subsequence $\left\{\left(H_{i}^{\prime}, S_{i}^{\prime}\right)\right\}$ such that the condition holds. Then by continuity of inducing (Theorem 1.2 of [3]),

$$
\left(H_{i}^{\prime}, \operatorname{ind}_{H_{i}^{\prime \prime}}^{H_{\prime}^{\prime}}\left(T_{i}\right)\right) \rightarrow\left(H, \operatorname{ind}_{H^{\prime \prime}}^{H}(T)\right) \text { in } \mathscr{Q}(G) .
$$

Since $\left(H_{i}^{\prime \prime}, T_{l}\right)$ is inducing for $\left(H_{i}^{\prime}, S_{i}^{\prime}\right)$, we have

$$
\operatorname{ind}_{H_{i}^{\prime \prime}}^{H_{i}^{\prime}}\left(T_{i}\right) \cong S_{i}^{\prime}
$$

for each $i$. Thus

$$
\left(H_{i}^{\prime}, S_{i}^{\prime}\right) \rightarrow\left(H, \operatorname{ind}_{H^{\prime \prime}}^{H}(T)\right) \text { in } \mathcal{Q}(G) .
$$

But $S \subseteq_{\mathrm{w}}$ ind $_{H^{\prime \prime}}^{H}(T)$, which implies

$$
\left(H_{i}^{\prime}, S_{i}^{\prime}\right) \rightarrow(H, S) \text { in } \mathbb{Q}(G) .
$$


Therefore,

$$
\left(H_{n}, S_{n}\right) \rightarrow(H, S) \text { in } \mathscr{Q}(G)
$$

3.5 Corollary. Let $\left\{\left(H_{n}, S_{n}\right)\right\}$ be a sequence in $\mathscr{Q}(G)$ such that $S_{n} \in \hat{H}_{n}$ and $S_{n}$ is not a character. Then $\left(H_{n}, S_{n}\right) \rightarrow(H, S)$ in $\mathbb{Q}(G)$ if and only if, for every subsequence of $\left\{\left(H_{n}, S_{n}\right)\right\}$, there exists a subsequence $\left\{\left(H_{i}^{\prime}, S_{i}^{\prime}\right)\right\}$ such that:

(1) There exist pairs $\left(K_{i}, \chi_{l}\right) \in \mathbb{Q}(G)$, where $\chi_{i}$ is a character of $K_{i}$ and $S_{l}^{\prime} \cong \operatorname{ind}_{K_{l}}^{H_{t}} \chi_{l}$, such that

$$
\left(K_{i}, \chi_{i}\right) \rightarrow(K, \chi) \text { in } \mathbb{Q}(G)
$$

where $\chi$ is a character of $K$.

(2) $S \subseteq_{\mathrm{w}} \operatorname{ind}_{K}^{H}(\chi)$

Proof. Let $\left(H_{n}, S_{n}\right) \rightarrow(H, S)$ in $\mathbb{Q}(G)$. By successively applying Theorem 3.4, and reducing to appropriate sequences, the sequence $\left\{\left(K_{i}, \chi_{i}\right)\right\}$ and the pair $(K, \chi)$ can be produced that satisfy the corollary. The opposite direction is shown in the same manner as in the proof of Theorem 3.4.

We can now use Theorem 3.4 to prove a lemma which clearly suffices to prove the Kirillov conjecture.

Lemma. Let $\left(H_{n}, S_{n}\right) \rightarrow(H, S)$ in $\mathbb{Q}(G)$. If $f \in \mathfrak{g}^{*}$ such that $\left.f\right|_{\mathfrak{h}} \in \Omega_{S}$, then for every subsequence of $\left\{\left(H_{n}, S_{n}\right)\right\}$, there is a subsequence $\left\{\left(H_{l}^{\prime}, S_{l}^{\prime}\right)\right\}$ such that for each $i$, there exists $f_{i} \in \mathrm{g}^{*}$ such that $\left.f_{l}\right|_{\mathfrak{h}_{i}^{\prime}} \in \Omega_{S_{l}^{\prime}}$ and $f_{l} \rightarrow f \in \mathrm{g}^{*}$.

Proof. Let $\left\{\left(H_{i}^{\prime}, S_{i}^{\prime}\right)\right\}$ be a subsequence of $\left\{\left(H_{n}, S_{n}\right)\right\}$ and restrict to a subsequence so that we may assume the dimensions of all $H_{t}^{\prime}$ are constant.

Let this constant be $k$. Then $\operatorname{dim} H=k$.

If each $S_{i}^{\prime}$ is a character of $H_{i}^{\prime}$, then $S$ is a character of $H$. In this case, it is easily seen that $\Omega_{S}$ and the $\Omega_{S_{i}^{\prime}}$ contain only one element.

Let $f \in \mathrm{g}^{*}$. By passage to another subsequence, we may assume there exist bases $h_{1}^{(l)}, \ldots, h_{n}^{(i)}$ of $\mathrm{g}$ such that $h_{1}^{(l)}, \ldots, h_{k}^{(l)}$ is a basis of $\mathfrak{h}_{l}, h_{l}^{(l)} \rightarrow h_{j}$ in $\mathrm{g}, 1 \leq j \leq n$, and $h_{1}, \ldots, h_{n}$ is a basis of $H$.

Define $f_{l} \in \mathrm{g}^{*}$ by

$$
f_{l}\left(h_{j}^{(i)}\right)= \begin{cases}f_{l}^{\prime}\left(h_{J}^{(i)}\right), & j \leq k, \\ f\left(h_{J}\right), & j>k,\end{cases}
$$

where $f_{t}^{\prime}$ is the singleton element of $\Omega_{S_{i}^{\prime}}$. 
Clearly $f_{t} \rightarrow f$ in $\mathrm{g}^{*}$ and the result follows.

The lemma proceeds by induction on $k$. If $k=1$, then each $S_{l}^{\prime}$ is a character and we are done. Assume, then, that the lemma has been shown for all sequences $\left\{\left(H_{i}^{\prime}, S_{l}^{\prime}\right)\right\}$ whose first elements all have dimension less than $k$.

By the above and by passage to a subsequence, we may assume each $S_{i}^{\prime}$ is not a character of $H_{i}^{\prime}$. By Theorem 3.4 there exist inducing pairs $\left(H_{n}^{\prime \prime}, T_{n}\right)$ for each $\left(H_{n}^{\prime}, S_{n}^{\prime}\right)$ such that

$$
\left(H_{i}^{\prime \prime}, T_{n}\right) \rightarrow\left(H^{\prime \prime}, T\right) \text { in } \mathbb{Q}(G)
$$

for some $T \in \hat{H}^{\prime \prime}$.

Now using the inductive hypothesis there exist $f_{i} \in \mathrm{g}^{*}$ such that $\left.f_{i}\right|_{\mathfrak{h}_{i}^{\prime \prime}} \in \Omega_{T_{i}}$ for each $i$, and $f_{l} \rightarrow f$ in $\mathfrak{g}^{*}$. But since $\left(H_{i}^{\prime \prime}, T_{l}\right)$ is inducing for $\left(H_{i}^{\prime}, S_{i}^{\prime}\right),\left.f_{i}\right|_{\mathfrak{H}^{\prime}} \in \Omega_{S_{i}^{\prime}}$ and the desired sequence is constructed.

Given this lemma, the fact that the Kirillov correspondence is a homeomorphism is straightforward.

3.7 THEOREM (Kirillov-Brown). $W_{n} \rightarrow W$ in $\hat{G}$ if and only if $\Omega_{W_{n}} \rightarrow \Omega_{W}$ in the space of orbits equipped with the quotient topology.

Proof. If $W_{n} \rightarrow W$ the theorem follows directly from Lemma 3.8. The opposite direction has been shown by Kirillov [4].

\section{REFERENCES}

[1] I. Brown, Dual topology of a nilpotent Lie group, Ann. Scient. Ec. Norm Sup., (4) 6 (1973), 407-411.

[2] J. M. G. Fell, Weak containment and induced representations of groups, Canad. J. Math., 14 (1962), 237-268.

[3] _ Weak containment and induced representations. II, Trans. Amer. Math. Soc., 110 (1964), 424-447.

[4] A. A. Kirillov, Unitary representations of nilpotent Lie groups, Russ. Math. Survey, 17 (1962), 53-104.

[5] G. W. Mackey, Induced representations of locally compact groups. I, Ann. of Math., (2) 55 (1952), 101-139.

[6] __ Unitary Representations of Group Extension. I, Acta Math., 99 (1958), 265-311.

Received November 19, 1982.

UNIVERSITY OF CALIFORNIA

DAVIS, CA 95616 



\section{PACIFIC JOURNAL OF MATHEMATICS \\ EDITORS}

DONALD BABBITT (Managing Editor)

University of California.

Los Angeles, CA 90024

Hugo Rossi

University of Utah

Salt Lake City, UT 84112

C. C. Moore and Arthur Ogus

University of California

Berkeley, CA 94720
J. DugundjI

Department of Mathematics

University of Southern California

Los Angeles, CA 90089-1113

R. FinN and H. Samelson

Stanford University

Stanford, CA 94305

\section{ASSOCIATE EDITORS}
R. ARENS
E. F. BECKENBACH
B. H. NeumanN
F. WOLF
K. YoshidA (1906-1982)

\section{SUPPORTING INSTITUTIONS}

UNIVERSITY OF ARIZONA

UNIVERSITY OF BRITISH COLUMBIA

CALIFORNIA INSTITUTE OF TECHNOLOGY

UNIVERSITY OF CALIFORNIA

MONTANA STATE UNIVERSITY

UNIVERSITY OF NEVADA. RENO

NEW MEXICO STATE UNIVERSITY

OREGON STATE UNIVERSITY
UNIVERSITY OF OREGON

UNIVERSITY OF SOUTHERN CALIFORNIA

STANFORD UNIVERSITY

UNIVERSITY OF HAWAII

UNIVERSITY OF TOKYO

UNIVERSITY OF UTAH

WASHINGTON STATE UNIVERSITY

UNIVERSITY OF WASHINGTON 


\section{Pacific Journal of Mathematics}

Vol. 112, No. $1 \quad$ January, 1984

Richard Blaine Barrar and Henry Loeb, Characterizing the divided difference weights for extended complete Tchebycheff systems $\ldots \ldots \ldots \ldots 1$

Harold Bennett and David John Lutzer, Generalized ordered spaces with

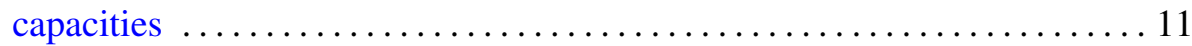

Geoffrey J. Butler and Lynn Harry Erbe, Comparison theorems for second-order operator-valued linear differential equations

Bohumil Cenkl and Richard D. Porter, de Rham theorem with cubical

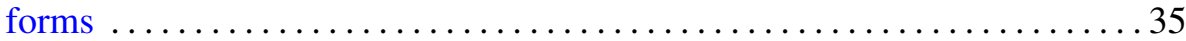

Zvonko Cerin, Characterizing global properties in inverse limits . ....... 49

Thomas Eugene Duchamp and Morris Kalka, Holomorphic foliations and deformations of the Hopf foliation .........................69 69

John Paul Hempel, Homology of coverings $\ldots \ldots \ldots \ldots \ldots \ldots \ldots \ldots \ldots$

Gerald Norman Hile and R. Z. Yeh, Inequalities for eigenvalues of the

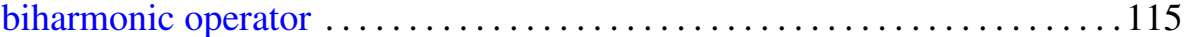

Kenneth Irwin Joy, A description of the topology on the dual space of a

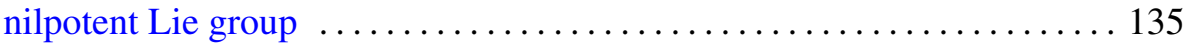

Alex Kumjian, On localizations and simple $C^{*}$-algebras $\ldots \ldots \ldots \ldots \ldots 141$

Bernardus de Pagter, The space of extended orthomorphisms in a Riesz

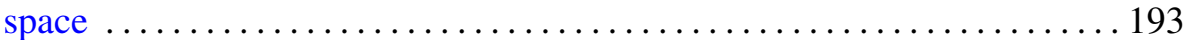

Stephen C. Persek, Iterated averaging for periodic systems with hidden

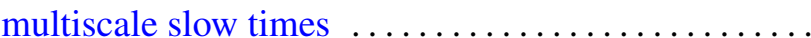

David Francis Rearick, Divisibility of arithmetic functions . . . . . . . . . 237

Masaaki Suzuki, The intrinsic metrics on the circular domains in $\mathbf{C}^{n}$ 